\title{
Shared decision making for the initiation and continuation of dialysis: a proposal from the Japanese Society for Dialysis Therapy
}

\author{
Kazuyoshi Okada 1* , Ken Tsuchiya ${ }^{2}$, Ken Sakai ${ }^{3}$,Takahiro Kuragano ${ }^{4}$, Akiko Uchida ${ }^{5}$, Kazuhiko Tsuruya ${ }^{6}$, \\ Tadashi Tomo ${ }^{7}$, Chieko Hamada ${ }^{8}$, Masafumi Fukagawa ${ }^{9}$, Yoshindo Kawaguchi ${ }^{10}$, Yuzo Watanabe ${ }^{11}$, \\ Kaoruko Aita ${ }^{12}$, Yoshitatsu Ogawa ${ }^{13}$, Junji Uchino ${ }^{14}$, Hirokazu Okada ${ }^{15}$, Yutaka Koda ${ }^{16}$, Yasuhiro Komatsu ${ }^{17}$, \\ Hisamitsu Sato ${ }^{18}$, Motoshi Hattori ${ }^{19}$, Toru Baba ${ }^{20}$, Mamiko Matsumura ${ }^{21}$, Hisayuki Miura ${ }^{22}$, Jun Minakuchi ${ }^{1}$, \\ Hidetomo Nakamoto ${ }^{23}$ and on behalf of Committee for Updating the Proposal for Shared Decision Making on \\ Initiation and Continuation of Dialysis, General Affairs Committee of the Japanese Society for Dialysis Therapy
}

\begin{abstract}
Background: In Japan, forgoing life-sustaining treatment to respect the will of patients at the terminal stage is not stipulated by law. According to the Guidelines for the Decision-Making Process in Terminal-Stage Healthcare published by the Ministry of Health, Labor and Welfare in 2007, the Japanese Society for Dialysis Therapy (JSDT) developed a proposal that was limited to patients at the terminal stage and did not explicitly cover patients with dementia. This proposal for the shared decision-making process regarding the initiation and continuation of maintenance hemodialysis was published in 2014.

Methods and results: In response to changes in social conditions, the JSDT revised the proposal in 2020 to provide guidance for the process by which the healthcare team can provide the best healthcare management and care with respect to the patient's will through advance care planning and shared decision making. For all patients with end-stage kidney disease, including those at the nonterminal stage and those with dementia, the decision-making process includes conservative kidney management.
\end{abstract}

Conclusions: The proposal is based on consensus rather than evidence-based clinical practice guidelines. The healthcare team is therefore not guaranteed to be legally exempt if the patient dies after the policies in the proposal are implemented and must respond appropriately at the discretion of each institution.

Keywords: Shared decision making, Advance care planning, Chronic kidney disease, End-stage kidney disease, Dialysis, Fogo

*Correspondence: kokada@khg.or.jp

${ }^{1}$ Department of Nephrology, Kawashima Hospital, 1-39 Kitasakoichiban-cho, Tokushima-shi, Tokushima 770-0011, Japan

Full list of author information is available at the end of the article

\begin{abstract}
Background
In discussing and making decisions about healthcare management and care options, it is important to respect the perspectives of patients until the final moments of their lives. The Ministry of Health, Labour and Welfare (MHLW) of Japan recognizes the importance of such respect, and accordingly, the Guidelines for the Decision-Making Process in Terminal-Stage Healthcare
\end{abstract} original author(s) and the source, provide a link to the Creative Commons licence, and indicate if changes were made. The images or other third party material in this article are included in the article's Creative Commons licence, unless indicated otherwise in a credit line to the material. If material is not included in the article's Creative Commons licence and your intended use is not permitted by statutory regulation or exceeds the permitted use, you will need to obtain permission directly from the copyright holder. To view a copy of this licence, visit http://creativecommons.org/licenses/by/4.0/. The Creative Commons Public Domain Dedication waiver (http://creativeco mmons.org/publicdomain/zero/1.0/) applies to the data made available in this article, unless otherwise stated in a credit line to the data. 
(formulated in 2007) [1] were renamed as the Guidelines for the Decision-Making Process in End-of-Life Healthcare [2] in 2015. Then, in 2018, to reflect the aging of the population and the accompanying increase in deaths in Japan, further revisions were made and published as the Guidelines for the Decision-Making Process in End-ofLife Healthcare Management and Care [3]. These guidelines note the need to establish an integrated community care system that incorporates the concepts of shared decision making (i.e., the process of choosing the best option for patients through repeated discussions) and advance care planning (i.e., the process through which patients make decisions about their future healthcare through repeated discussions with their family members or other relevant persons (hereafter, "families") and healthcare teams) into healthcare and end-of-life (EOL) care. Given that patients may change their decisions or may become unable to communicate their decisions, the guidelines highlight that trusted individuals (e.g., family members) who can infer patients' decisions should be involved in discussions on healthcare management and care strategies so that patients can live until their final moments with their dignity respected and upheld.

Approximately 2 years after the 2014 publication of the Proposal for the Shared Decision-Making Process Regarding the Initiation and Continuation of Maintenance Hemodialysis [4], a nationwide survey [5] revealed that $47.1 \%$ of dialysis facilities had cases where a temporary decision to forgo dialysis was made that allowed for the later initiation or reinitiation of dialysis depending on changes in the patient's decision or disease status. This temporary approach is widely known as "Miawase" in Japanese, and notably, the "Miawase" approach is different from permanently forgoing (withdrawing or withholding) and discontinuing dialysis.

In a nationwide survey, among patients who opted for the temporary decision to forgo dialysis, $89.7 \%$ were elderly, $46.1 \%$ had dementia, and $7.5 \%$ later initiated or reinitiated dialysis. Among these patients, $23.4 \%$ did not meet the criteria for a temporary decision established in the proposal, but this approach was selected based on the firm decisions made by these non-EOL patients with their families' agreement, which indicates the difficult situations in which healthcare teams often need to make judgments [5].

With respect to dementia, the Clinical Practice Guidelines for Dementia 2017 were published by the Japanese Society of Neurology [6], and the Guidelines for Supporting Decision Making in Daily and Social Life for Individuals with Dementia were published by the MHLW in 2018 [7]. Humans have intentions and desires even when their cognitive function is impaired; thus, patients' decisions should be respected, and their decision making should be supported based on the appropriate assessment of their decision-making capacity. The MHLW guidelines systematically describe basic concepts, attitudes, approaches, and matters to be considered by people who closely support decision making by individuals with impaired cognitive function so that they can live their daily and social lives according to own their decisions.

Because the previous proposal was limited to patients on maintenance hemodialysis at the terminal stage and did not explicitly cover patients with dementia, revisions have subsequently been made so that the proposal applies to a broader range of patients, can help healthcare teams provide better healthcare management and care, and are suitable for the current healthcare environment. The patients for whom the proposal is intended to be applicable include those on peritoneal dialysis and those at the initiation phase of hemodialysis for end-stage kidney disease (ESKD) or acute kidney disease, those at the terminal stage, and those with dementia. This proposal describes in detail shared decision making and the adequate implementation of advance care planning. The aim is for dialysis facilities across Japan to use this proposal as a suitable reference and for healthcare teams to respect patients' decisions, take their intentions into account, and thereby support them in leading a dignified life with their wishes fulfilled until their final moments.

This proposal describes the process of how healthcare teams can provide information about renal replacement therapy (RRT) and conservative kidney management (CKM) as medical care options for patients with ESKD [8] and healthcare teams' decision-making processes regarding the temporary decision to forgo dialysis when patients and their families opt for CKM. The determination of the implementation process of this proposal is left to each facility.

\section{Withholding and withdrawal of dialysis in Japan and overseas}

The withholding of dialysis and the withdrawal of dialysis are collectively described as forgoing dialysis. The withdrawal means that patients who have undergone dialysis stop dialysis, and the withholding means that patients do not start dialysis. Outside Japan, in 1986, cases of dialysis withdrawal were expected to increase as patients on dialysis aged [9], and in 1994, a list of conditions for which the withholding of maintenance dialysis was to be suggested to patients and their families was created [10].

Since the 1990s, in the United States, respecting patients' decision-making rights and recognizing the importance of shared decision making and advance care planning have been key to the withholding and withdrawal of dialysis. Therefore, in 2000, the Renal Physicians Association and the American Society of 
Nephrology published Shared Decision-Making in the Appropriate Initiation of and Withdrawal from Dialysis: Clinical Practice Guideline [11], which was later revised in 2010 [12].

There was a similar movement in Japan as well [13], leading to the publication of the Proposal for the Shared Decision-Making Process Regarding the Initiation and Continuation of Maintenance Hemodialysis by the Japanese Society of Dialysis Therapy (JSDT) in 2014 [4]. Recently, in the field of dialysis, shared decision making has been widely adopted when RRT and/or a temporary decision to forgo dialysis are options and when advance care planning is conducted for EOL healthcare management and care after the temporary decision to forgo dialysis is terminated.

According to 2000-2015 statistics from the United States Renal Data System, deaths due to the withdrawal of dialysis accounted for $19 \%$ of the total deaths in 2000 , peaked in 2011 (25\% of the total), and remained high in 2015 (23\% of the total, the third-largest cause of death after cardiovascular diseases and infections; latest time point of available data), although there were variations by race, age, sex, and region [14]. A 12-year observational study of 867 patients in the United Kingdom showed that 93 patients (11\%) opted for the withdrawal of dialysis in the decision making on EOL [15]. The number of patients opting for the withdrawal of dialysis increased from 3 per 1000 patient-years in 1966 to 48.6 per 1000 patient-years in 2010 [16].

As proposed in guidelines published by the Oxford University Hospital Kidney Unit in 2018 [8], CKM should be performed even after the decision to withhold or withdraw dialysis has been made. In a recent study in Canada, CKM was reported to be a medical care option for stage 5 chronic kidney disease (CKD); CKM was described as involving recognition of the value of quality of life (QOL), active symptom management, and advance care planning; the implementation of shared decision making; the provision of patient-centered care; and the inclusion other interventions to delay the progression of ESKD, but not dialysis [17]. The content of CKM can vary, but it is roughly classified into measures for managing the complications of CKD (e.g., hypertension and electrolyte imbalance) and measures for managing symptoms (vomiting and pruritus), both of which involve pharmacologic and nonpharmacologic interventions (e.g., lifestyle interventions, environmental interventions, and adjuvant therapy). These measures should be planned and adjusted based on clinical assessments and advance care planning. Although CKM is becoming recognized as an appropriate medical care option for patients who are unlikely to benefit from dialysis or those who opt to withhold dialysis, there are great variations in the conceptualization and implementation of CKM $[17,18]$. Even in the United Kingdom, where CKM is relatively well accepted, its content and intensity vary considerably [18].

In Asia, a study in Hong Kong published in 2004 reported on 107 patients who decided to withhold or discontinue dialysis and receive palliative care [19]. Taiwan was the first Asian country to make doctors exempt from legal liability for terminating life-sustaining medical care; subsequently, consensus on withholding and withdrawal of dialysis was reached, and active care planning was mentioned in the law in 2011, which noted the importance of promoting palliative care and home-based healthcare when dialysis is withheld or withdrawn [20]. Such changes during the 2000s in neighboring Asian countries likely influenced the discussions that would happen in Japan.

Several studies have compared CKM and RRT in patients who opted to withhold or withdraw dialysis. An Australian cohort study showed that some patients who opted for CKM survived for $\geq 3$ years and that patients who opted for CKM were more likely than those who received dialysis to have palliative care and to die at home or in hospice rather than in the hospital [21]. A single-center study from the Netherlands showed a more favorable prognosis with dialysis than with CKM in patients aged $\geq 70$ years, but the difference became negligible in patients aged $\geq 80$ years and in patients with a cardiovascular comorbidity [22]. These results show that the prognosis after appropriate CKM may be as favorable as that after dialysis. According to recent systematic reviews of studies comparing CKM with RRT, the survival rate was found to be significantly higher with RRT than with CKM [23], but the QOL of patients with CKM tended to be better than, or at least similar to, that of patients with RRT [24, 25].

However, in the interpretation of these data from Europe and North America, it should be noted that patients were given information about four medical care options for ESKD, including RRT and CKM, to choose treatment at an early stage when the estimated glomerular filtration rate (eGFR) was approximately $15 \mathrm{~mL} / \mathrm{min} / 1.73 \mathrm{~m}^{2}$. Thus, the results on the comparison of prognoses are not directly applicable to Japanese patients. In Japan, a multidisciplinary healthcare team approach, including nonpharmacologic therapy, has been introduced to avoid dialysis and RRT, but the content of care in CKM after the decision to forgo dialysis still needs to be established in collaboration with home-based healthcare doctors. To accomplish this, differences in patients' autonomy, family relationships, social security system, religious beliefs, and dialysis therapy must be taken into consideration. 


\section{Commentary on the individual proposals}

Proposal 1. Respect for patients' decision making by healthcare teams ${ }^{*}$ 1. Respect patients' decisions about healthcare management and care strategies

2. Obtain a consent form for the initiation of dialysis from patients before initiating dialysis

3. Provide information to patients about their right to prepare advance directives ${ }^{*}$.3. Provide information to patients about their right to prepare advance directives ${ }^{* 2}$

\section{Commentary}

1. Respect patients' decisions about healthcare management and care strategies

Patients with decision-making capacity have the right to receive clear and appropriate information and to accept or decline healthcare treatment based on their own decisions, regardless of whether they are in the EOL stage. Healthcare teams should understand this right and respect patients' decisions about healthcare management and care strategies. If patients do not currently have decision-making capacity but previously left instructions on healthcare management and care strategies (written or oral) when they had decision-making capacity and if consensus is reached with the families, healthcare teams should respect those healthcare management and care strategies. If consensus is not reached, healthcare teams should repeatedly provide information to patients' families about the need to respect patients' decisions so that consensus is reached.

2. Obtain a consent form for the initiation of dialysis from patients before initiating dialysis

Patients can choose their medical care. It is advisable for healthcare teams to clearly provide the necessary information to patients so that they understand it before making their decisions. If patients have decision-making capacity, a consent form needs to be obtained from them in the presence of their families before dialysis is initiated. If patients do not have decision-making capacity, their families have no choice but to become surrogate decision makers about their healthcare management and care, and thus, a consent form must be obtained from them. In this case, it is advisable that healthcare teams clearly provide the necessary information to families so that they understand it and can then make surrogate decisions.

When a consent form cannot be obtained because families are unable to come to healthcare facilities, healthcare teams should make a note in patients' medical documents about the content of information given to patients' families and what they consented to over the phone. When families are unreachable, healthcare teams should record in patients' medical documents what information would have been provided to them and should preferably obtain a consent form from the families after they have provided that information. Healthcare teams should also record in patients' medical documents what the patients' families consented to.

If it is impossible to obtain a consent form from a suitable person other than the patient, healthcare teams should make a note in the patient's medical documents about the reasons, such as that the patient had no relatives or friends, the patient lived alone and his or her entry in the family registry was unknown, or that relatives did not wish to engage in any patient-related communication.

3. Provide information to patients about their right to prepare advance directives $^{* 2}$

If patients have not yet prepared advance directives, healthcare teams should inform them that they have the right to prepare advance directives and that, based on the directive, healthcare teams can understand patients' intended decisions and provide them with their preferred healthcare management and care at any time under any conditions in the future. Additionally, healthcare teams should inform patients that an advance directive should be routinely reviewed and can be withdrawn at any time.

Proposal 2. Shared decision making with patients

1. Provide patients with adequate information

2. Collect adequate information from patients

3. Have thorough discussions to support patients in making the best possible choices

4. Provide patients with adequate information about renal replacement therapy (RRT)

(1) At the appropriate time, provide information about RRT becoming necessary when kidney function deteriorates in the future

(2) For patients for whom dialysis will become necessary in the near future, provide information about RRT and the natural course of endstage kidney disease (ESKD)

5. If patients do not opt for RRT when dialysis needs to be initiated, have repeated discussions with patients and their family members ${ }^{* 3}$ (including heirs ${ }^{*}$ ) for consensus building

(1) Continue discussions until the advantages and disadvantages of conservative kidney management (CKM) and the initiation of dialysis are understood

(2) Have discussions in accordance with the decision-making process (Fig. 1)

(3) When patients make a final decision to opt for CKM, obtain a confirmation form for the "Miawase" approach to dialysis*5 if necessary

(4) Reassess patients for their changes in decisions when they visit healthcare facilities

6. Assess whether patients are receiving healthcare management and care in compliance with the decisions they have made 


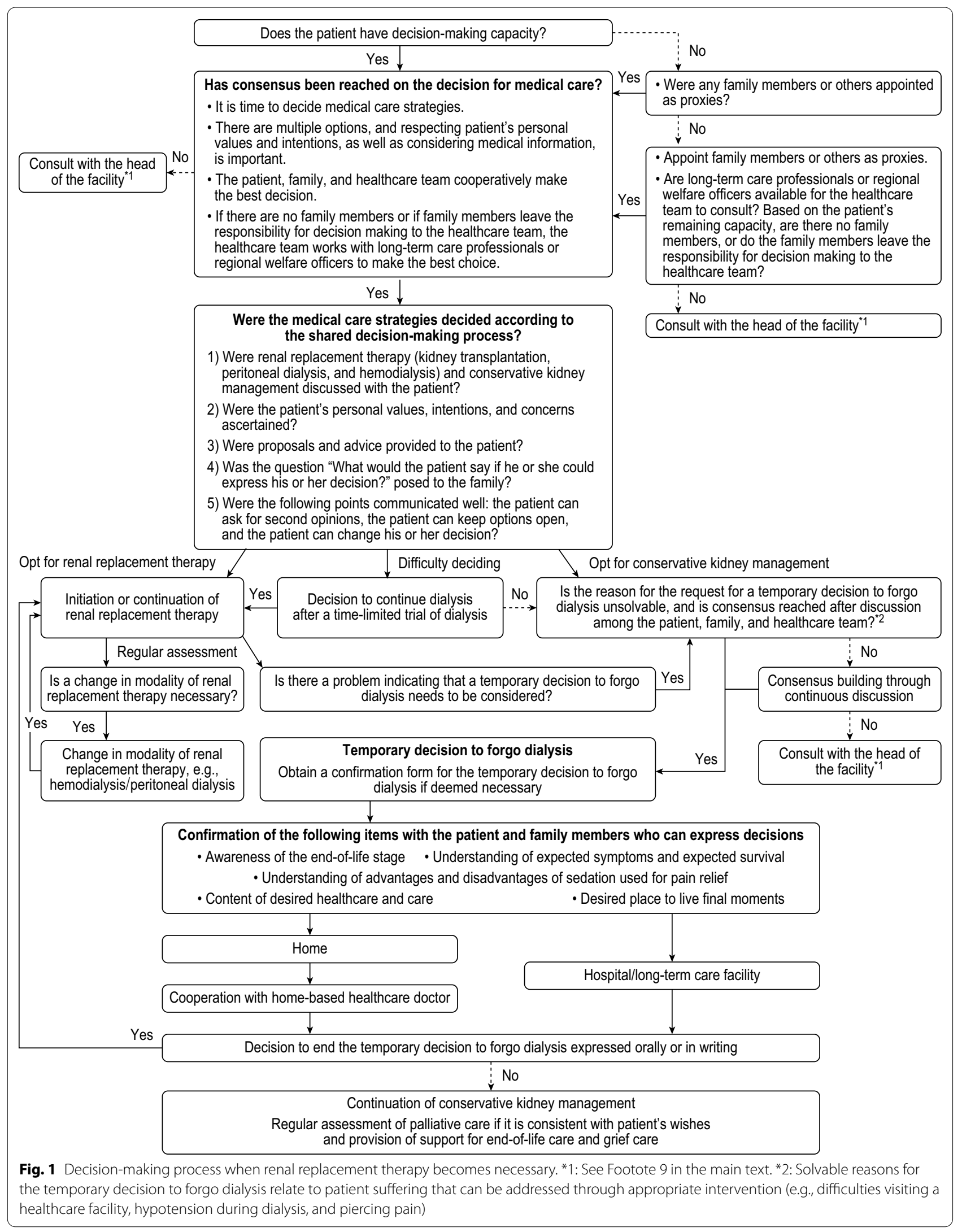




\section{Commentary}

1. Provide patients with adequate information

It is advisable for medical care strategies and other care plans to be decided through a shared decision-making process. During shared decision making, healthcare teams should provide information in understandable plain language about the diagnosis of CKD, complications, prognosis, advantages and disadvantages of various interventions (lifestyle improvement, diet therapy, exercise therapy, and pharmacotherapy), and their influences on daily life.

2. Collect adequate information from patients

Healthcare teams should listen to patients to determine their level of understanding. At the same time, healthcare teams should collect information about patients' personal values, intentions, interests, motivations, concerns, unclear points, financial uncertainty, living environment, relationships with relatives, stress, and preferred healthcare management and care.

3. Have thorough discussions to support patients in making the best possible choices

Through effective communication, healthcare teams can help patients improve their resilience against disease and answer patients' questions until they accurately understand their disease status and the risks and benefits of each medical care option. Healthcare teams should understand patients' personal values and preferences, propose medical care options that are consistent with patients' interests, and thereby aim to reach consensus.

The best choice made by patients may be different from ideas about the best possible healthcare management and care from the viewpoints of medicine, nursing, and longterm care. Healthcare teams should support patients in making the best choice that is consistent with their personal values based on a good awareness of other parties' opinions and an accurate understanding of all the information provided.

4. Provide patients with adequate information about renal replacement therapy (RRT)

(1) At the appropriate time, provide information about RRT becoming necessary when kidney function deteriorates in the future

(2) For patients for whom dialysis will become necessary in the near future, provide information about RRT and the natural course of endstage kidney disease (ESKD)

When the eGFR becomes less than $30 \mathrm{~mL} / \mathrm{min} / 1.73 \mathrm{~m}^{2}$ with progressive deterioration of kidney function, healthcare teams should provide patients with information related to RRT (kidney transplant, peritoneal dialysis, and hemodialysis) that will become necessary when kidney function further deteriorates in the future.

When RRT is expected to be initiated in the near future, healthcare teams should provide patients with RRT-related information, explain the natural course of ESKD, and discuss the information with them until they understand the advantages and disadvantages of opting or not opting for RRT. It is important that patients understand their situations and live their lives. If patients are unsure about decision making, healthcare teams should provide them with support when they visit healthcare facilities.

5. If patients do not opt for RRT when dialysis needs to be initiated, have repeated discussions with patients and their family members ${ }^{*}$ (including heirs ${ }^{*}$ ) for consensus building

(1) Continue discussions until the advantages and disadvantages of conservative kidney management (CKM) and the initiation of dialysis are understood

(2) Have discussions in accordance with the decision-making process (Fig. 1)

(3) When patients make a final decision to opt for CKM, obtain a confirmation form for the "Miawase" approach to dialysis*5 if necessary

(4) Reassess patients for changes in their decisions when they visit healthcare facilities

It is important for healthcare teams to understand all aspects of patients' lives and their anxieties and problems and, through discussions with patients and their families, to identify measures that align with the patients' interests so that all parties involved are satisfied. If patients have mental and/or social problems, it is important to consider appropriate interventions. In the consensus-building process, healthcare teams should provide information about the disease status and other relevant information, propose measures necessary for patients to live their lives with dignity, and have discussions to help patients accept that such proposals are required as necessary in a comprehensible and professional manner.

If RRT has not yet been implemented when kidney disease reaches the end stage and the initiation of life-sustaining dialysis is required, patients then face a decision-making situation regarding RRT. Healthcare teams should provide the following information to help patients in their decision making: the timing of RRT initiation and modality options (kidney transplantation, peritoneal dialysis, or hemodialysis) if opting for RRT; the natural course of the disease if not opting for RRT; and the advantages and disadvantage of opting for and not opting for RRT. When patients request a nonRRT option, healthcare teams should provide information about CKM and have discussions with patients and their families following the shared decision-making process. If CKM is chosen by patients and their families and the Japanese "Miawase" approach to dialysis (the 
temporary decision to forgo dialysis) is implemented, healthcare teams should provide information on the following possibilities until patients and their families fully understand them: death due to uremic symptoms (e.g., dyspnea, disturbance of consciousness, pulmonary edema, and arrhythmia); avoidance of severe uremic symptoms through the initiation of dialysis; suffering associated with dialysis; reduction in suffering through palliative care; initiation or continuation of dialysis by withdrawing the temporary decision to forgo dialysis; the possibility of death even after the initiation of dialysis; and time-limited trial of dialysis. When patients' decision-making capacity is in question due to their mental conditions or other reasons, healthcare teams should encourage patients and their families to visit specialists.

If patients undergo a shared decision-making process and eventually choose CKM, a confirmation form for the temporary decision to forgo dialysis should be obtained from patients and their families if deemed necessary. It is important that all professionals in healthcare teams record the content of discussions with patients and their families, prepare summary reports, and share them with patients and their families. Patients' decisions need to be reviewed depending on changes in disease status even after confirmation forms are provided. An example of a confirmation form is provided in the Additional file 1. Obtaining a confirmation form is not mandatory and is sometimes omitted to respect patients' decisions. It is also important to provide patients' family members with psychological care, for example, at the EOL phase, and grief care ${ }^{* 6}$.

If patients visit healthcare facilities regularly even after choosing CKM, healthcare teams should assess their disease status, provide necessary palliative care, and confirm whether they have made any changes to their decisions. If patients have changed their decisions and now request the initiation of dialysis, healthcare teams should initiate dialysis according to the oral instruction provided by patients, discuss healthcare management and care strategies again with patients and their families, and obtain a confirmation form for the withdrawal of the decision if deemed necessary.

Patients have the right to seek second opinions, and information about this right should be provided to patients.

6. Assess whether patients are receiving healthcare management and care in compliance with the decisions they have made

After patients have made decisions about healthcare management and care strategies, it is important to assess whether they are receiving the healthcare management and care they wish to receive. Healthcare teams should also assess the presence of anxiety associated with the temporary decision to forgo dialysis, the presence of symptoms due to disease progression, and whether appropriate care is provided for such anxiety and symptoms.

\footnotetext{
Proposal 3. Advance care planning with patients

1. Have thorough discussions on various occasions about future healthcare management and care

2. Have thorough discussions on patients' preferred healthcare management and care in accordance with the decision-making process (Fig. 1)

(1) Provide patients with information about the expected symptoms and prognosis after implementing the temporary decision to forgo dialysis

(2) When patients choose to have their final moments at home, cooperate with the doctors responsible for home-based healthcare

(3) Assess patients' changes in their decisions in response to changes in disease status
}

\section{Commentary}

1. Have thorough discussions on various occasions about future healthcare management and care

Advance care planning is a patient-centered discussion process in which patients, irrespective of their age and disease stage, share their personal values and life goals with their families and healthcare teams, have opportunities to consider and make decisions about healthcare management and care at EOL before their decision-making capacity is impaired, and receive support in decision making from their families and healthcare teams.

It is important that healthcare teams start advance care planning at the appropriate time so that patients can promptly receive healthcare management and care that is consistent with their decisions. Advance care planning needs to be implemented carefully when dialysis is initiated or when the patient's disease status changes.

2. Have thorough discussions on patients' preferred healthcare management and care in accordance with the decision-making process (Fig. 1)

(1) Provide patients with information about the expected symptoms and prognosis after implementing the temporary decision to forgo dialysis

(2) When patients choose to have their final moments at home, cooperate with the doctors responsible for home-based healthcare

(3) Assess patients' changes in their decisions in response to changes in disease status

Healthcare teams should participate in the decisionmaking process (Fig. 1) to understand patients' perspectives on life, personal values, wishes, and so forth and to recognize patients' priorities through thorough discussions with them and their families. In this way, health 
Table 1 Conditions when the temporary decision to forgo dialysis should be considered

\author{
1. When there are difficulties in the safe performance of dialysis and the patient's life is at risk \\ (1) The patient is in a condition where dialysis is harmful rather than beneficial to life because of multiple organ failure causing circulatory and respira- \\ tory problems, sustained hypotension, or other problems that make sustaining life extremely difficult \\ (2) The patient is in a condition where dialysis can be performed safely only with the use of physical restraint and sedation \\ 2. When the patient's general condition is extremely poor and his or her decision on the temporary decision to forgo dialysis is clearly expressed or \\ when family members can infer the patient's decision \\ (1) The patient is in a condition where serious cerebral dysfunction due to sequelae of cerebrovascular disease, head injury, etc., prevent him or her \\ from having the comprehension necessary to undergo dialysis and recuperation \\ (2) The patient is in a condition where death is imminent because of an incurable malignant comorbidity, such as a malignant tumor \\ (3) The patient is not capable of oral intake, and long-term life-sustaining artificial hydration and nutrition are expected
}

care teams can provide care that is consistent with patients' personal values and respect patients' dignity. Healthcare teams should provide healthcare management and care while fully respecting patients' decisions and should help patients live their lives with their dignity respected and upheld. Decisions should also be made about the discontinuation of low-priority pharmacotherapies and the transition from oral medications to injections or transdermal patches before these steps become necessary. When patients do not have decision-making capacity, such matters should be thoroughly discussed with their families.

It is important that healthcare teams provide patients and their families with information about the expected symptoms and prognosis after the temporary decision to forgo dialysis is terminated, provide care that reduces physical suffering as early as possible, and ensure the continuous provision of the necessary palliative care. For continuous deep sedation, it is advisable to obtain prior consent from patients or their families. It is also important that all professionals on healthcare teams record all remarks made by patients and their families during any decision-making discussions on healthcare management and care and to share written summaries with them after each discussion.

Patients' families should be involved in care for patients' overall pain (physical, psychological, social, and spiritual suffering"7). Healthcare teams provide palliative care to reduce physical pain, and they should listen to patients and their families to identify their psychological, social, and spiritual needs. It is advisable that palliative care specialists be involved as needed.

Healthcare teams should have discussions with patients and their families and strive to provide options that allow patients to spend time in their preferred places with their dignity maintained until their final moments. Healthcare teams should confirm patients' preferred places to be at the EOL, and if the home is chosen, teams should provide adequate support in cooperation with home-based healthcare doctors and long-term care professionals (e.g., care workers and care managers) so that patients can live their final moments with dignity.

Even after patients make their decisions, it is important to continue having discussions with them and their families to confirm any changes in decisions. If patients lose their decision-making capacity, the latest decisions they made while they still had such capacity should be followed.

Proposal 4. Proposal of the temporary decision to forgo dialysis at the EOL stage by healthcare teams

1. Judge the patient's condition regarding whether the temporary decision to forgo dialysis should be considered by referring to the Table 1

2. Engage in the decision-making process (Fig. 1)

3. Provide adequate palliative care after CKM is chosen and the temporary decision to forgo dialysis is implemented

\section{Commentary}

1. Judge the patient's condition regarding whether the temporary decision to forgo dialysis should be considered by referring to the Table 1

When the temporary decision to forgo dialysis is judged to be one of the best options for respecting patients' dignity at the EOL, healthcare teams should propose the approach to patients with decision-making capacity or to the families of patients who do not have such capacity and help them make decisions in accordance with the decision-making process. Healthcare teams should provide information about reducing the duration and frequency of dialysis therapy, as well as making the temporary decision to forgo dialysis.

2. Engage in the decision-making process (Fig. 1)

When patients with decision-making capacity wish to opt for a temporary decision to forgo dialysis, the decision should be discussed following the decision-making process. If consensus is not reached after discussions 
with patients and their families, healthcare teams should consult with the head of the facility to achieve consensus.

When patients with decision-making capacity cannot make a decision or are uncertain about their decisions, healthcare teams should provide appropriate support for their decision making upon request. Decisions made through such a process should be shared and respected.

When families of patients without decision-making capacity infer patients' decisions and opt for the temporary decision to forgo dialysis and when consensus is reached with healthcare teams, the process through which the families respected the patients' decisions should be shared and respected by the healthcare teams. Healthcare teams should take patients' remaining decision-making capacity into account when holding discussions.

When families of patients without decision-making capacity can infer patients' decisions to opt for the temporary decision to forgo dialysis but cannot or are reluctant to implement this decision, healthcare teams should support them. If consensus is reached with healthcare teams, the process should be shared and respected.

When the families of patients who do not have decision-making capacity cannot infer patients' intentions, healthcare teams and families should discuss healthcare management and care for these patients. If consensus is reached, the resulting decision should be respected and reported to the head of the respective facility.

When patients who have decision-making capacity, or the families of patients without decision-making capacity, leave decision-making responsibilities to healthcare teams, teams should perform assessments to determine the best possible healthcare management and care strategies, provide information about the results of assessments to patients and their families, and make efforts to reach consensus with them.

When there are no family members of a patient without decision-making capacity, in principle, healthcare teams should examine the validity and relevance of healthcare management and care, take the patient's residual capacity into account, consult with care professionals and regional welfare officers ${ }^{* 8}$ who know the patient, and then choose the options that align with the patient's best interests. If care professions and regional welfare officers are not available, the matters should be discussed with the head of the respective facility. When patients' heirs are traceable through family registration documents, the agreement of heirs is legally significant, so it is advisable to consider contacting them.
3. Provide adequate palliative care after CKM is chosen and the temporary decision to forgo dialysis is implemented

In accordance with Proposal 2, a confirmation form for the temporary decision to forgo dialysis should be obtained from patients and their families if deemed necessary, and patient-centered discussions about future healthcare management and care should be held with patients or their families in accordance with Proposal 3. Even after decisions are made, it is important to cooperate with families to confirm whether patients have changed their decisions. Palliative care should appropriately treat symptoms irrespective of the stage of CKD and is therefore necessary even before the temporary decision to forgo dialysis is made: appropriate palliative care should be continuously provided as dictated by the situation.

Proposal 5. Request for the temporary decision to forgo dialysis by patients with decision-making capacity or by the families of patients without decision-making capacity to healthcare teams

1. Confirm the decision through discussion with patients with decisionmaking capacity or through the examination of any prior instruction (written or oral) made by patients without decision-making capacity 2. When healthcare teams judge that patients are not at the EOL stage, ESKD that requires maintenance dialysis for survival should be diagnosed

(1) When CKM is chosen through the decision-making process (Figure) and when consensus is reached among the relevant parties, obtain a confirmation form for the temporary decision to forgo dialysis if necessary and continuously provide adequate palliative care

(2) When patients' decisions cannot be inferred or when consensus is not reached among the relevant parties, continue discussions to build consensus

(3) Reassess patients for changes in their decisions when they visit healthcare facilities

\section{Commentary}

1. Confirm the decision through discussion with patients with decisionmaking capacity or through the examination of prior instruction (written or oral) made by patients without decision-making capacity

Patients have the right to make a decision, and when they request the temporary decision to forgo dialysis, the reasons for that decision should be identified through adequate discussions, as described in Proposal 2. If patients whose life can be sustained through the initiation or continuation of dialysis opt for CKM and wish to make the temporary decision to forgo dialysis, it is advisable to obtain an advance directive from them after providing understandable written information on the advantages and disadvantages of medical care.

When families of patients without decision-making capacity request the temporary decision to forgo dialysis, 
healthcare teams consult any advance directives or oral instruction that was prepared or expressed when the patients had decision-making capacity and then confirm patients' decisions and reasons for those decisions.

Healthcare teams should try to understand patients' anxieties and various problems, have discussions with patients and their families so that patients are motivated to live, and thus identify measures aligned with patients' interests. It is important to consider specific interventions if patients have psychological and social problems.

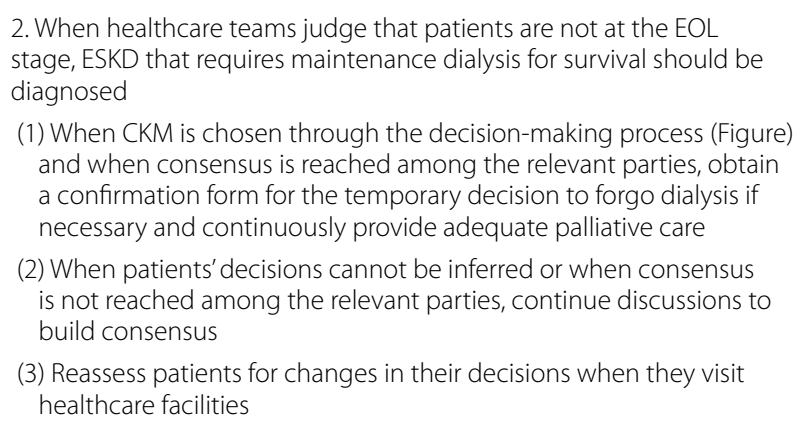

(1) When CKM is chosen through the decision-making process (Figure) and when consensus is reached among the relevant parties, obtain a confirmation form for the temporary decision to forgo dialysis if necessary and continuously provide adequate palliative care

(2) When patients' decisions cannot be inferred or when consensus is not reached among the relevant parties, continue discussions to build consensus

(3) Reassess patients for changes in their decisions when they visit healthcare facilities

Patients with decision-making capacity have the right to receive clear and appropriate information and the right to accept or decline healthcare based on their own decisions, regardless of whether they are in the EOL stage. For patients who are not yet in the EOL phase request the temporary decision to forgo dialysis, EOL is considered to begin when a physician diagnoses ESKD that requires maintenance dialysis as life-sustaining treatment. The patient's decision is the final decision at that time and must be respected. Healthcare teams must be aware that patients may change their decisions, and therefore, they must continuously be ready to initiate dialysis for them and to provide them with information about a time-limited trial of dialysis. Autonomy-that is, making one's own decisions about one's own affairs-is the most important element of human dignity. Thus, the decision to opt for CKM involving nondialysis interventions for ESKD must be respected when consensus is reached among the patient, the family, and the healthcare team following a thorough discussion based on appropriately shared essential information. Healthcare teams should obtain a confirmation form from patients and their families about the decision to opt for the temporary decision to forgo dialysis if it is deemed necessary and should provide palliative care that is consistent with patients' wishes. Moreover, palliative care is necessary even before this decision is made, and appropriate palliative care must be provided according to each patient's individual situation. When any healthcare team member from any profession discusses the selection of healthcare management and care with patients or their family members, the discussion must be documented, and a summary of the content must be shared with the patients and their family members.
Families of patients without decision-making capacity have no choice but to become surrogate decision makers regarding the patient's healthcare management and care. When families respect the patient's dignity by making a decision in accordance with his or her prior instruction and when consensus to opt for CKM is reached following adequate information sharing and repeated discussions among the family members and healthcare teams, the decision based on consensus should be respected. Healthcare teams should handle requests made by families similarly to how they handle requests made by patients with decision-making capacity: a confirmation form for the temporary decision to forgo dialysis should be obtained, if deemed necessary, and palliative care consistent with the patient's wishes should then be provided.

If families and healthcare teams cannot infer patients' decisions, healthcare teams should continue discussions so that family members can understand patients' conditions and thus provide the healthcare management and care deemed best for them. If consensus is reached, the decision should be respected and reported to the heads of facilities. If consensus is not reached, healthcare teams should consult with the heads of their facilities and work toward consensus building"9. Healthcare teams should consult with the heads of their facilities when they do not have sufficient time for repeated discussions.

Some predialysis patients with ESKD who make regular visits to healthcare facilities have strong feelings against dialysis and consequently wish to opt for CKM. For these patients, healthcare teams should evaluate their disease status, provide the necessary palliative care, and confirm whether they have changed their decisions.

If patients on dialysis do not visit healthcare facilities, healthcare teams should contact the patients to determine their reasons for not visiting, inform the families of the reasons, and proceed with the shared decisionmaking process as far as possible to reach a decision. When dialysis is declined, healthcare teams should inform patients and their families that the patients may die within a few weeks, if not a few days, and that they should contact healthcare facilities if they wish to reinitiate dialysis. If patients and their families are not reachable or if there are no family members, healthcare teams should consult with care workers and regional welfare officers about the situation and consider contacting the police to confirm patient safety.

Proposal 6. Requests from patients that their family, etc., not be notified about their disease status

1. Determine the reasons why patients do not want their families to be notified and evaluate patients' decision-making capacity

2. Refrain from contacting families if patients have decision-making capacity, but contact them if they do not have decision-making capacity 
3. When uremic symptoms are confirmed or when CKM is chosen and the temporary decision to forgo dialysis is to be implemented, inform patients that their family members will be contacted and then provide them the information about the disease status

\section{Commentary}

1. Determine the reasons why patients do not want their families to be notified and evaluate patients' decision-making capacity

The patient does not want to trouble families or those are not decision-making supporters for patient. Healthcare teams should identify the reasons for such situations. Additionally, healthcare teams should assess patients' decision-making capacity and confirm whether there are significant persons other than family members who can become involved.

2. Refrain from contacting families if patients have decision-making capacity, but contact them if patients do not have decision-making capacity

When it is appropriate for information about disease status to be provided to patients with decision-making capacity, there is no legal obligation to give further information to families if the patients are not in critical condition. However, if patients do not have decision-making capacity, information about the disease status should be provided to families after patients have been informed of their disease status.

3. When uremic symptoms are confirmed or when CKM is chosen and the temporary decision to forgo dialysis is to be implemented, inform patients that their family members will be contacted, and then contact them to provide information about the disease status

Given the social background in Japan and the cooperation and consideration that families are expected to provide after they receive information, if the patient's disease status is critical, family members should be provided with this information after patients have been informed of their disease status, even if patients request that their families not be contacted.

Proposal 7. Support for decision making by patients with impaired comprehension and cognition by healthcare teams and patients' families

1. Respect and support patients' decision making and provide them with the best possible healthcare management and care that are consistent with their wishes

2. Encourage patients' families to engage in advance care planning with patients while they still have decision-making capacity

\section{Commentary}

1. Respect and support patients' decision making and provide them with the best possible healthcare management and care management that are consistent with their wishes
Healthcare teams, in collaboration with patients' families, take care of patients with impaired comprehension and cognition so that they can live their lives with dignity, and they respect the decisions (intentions, preferences or tendencies) expressed by patients in compliance with the MHLW's Guidelines for Supporting Decision Making in Daily and Social Life for Individuals with Dementia. Based on the premise that patients intend to make decisions and have decisionmaking capacity regardless of their comprehension and cognition levels, healthcare teams should help patients make and express their decisions according to their conditions and should implement healthcare management and care that are aligned with their decisions.

Decision-making capacity can vary depending on psychosocial, environmental, medical somatic, psychological, and neurological conditions, as well as the levels of comprehension and cognition. Patients' decision-making capacity can change, and therefore, healthcare teams should support them according to their current decision-making capacity. To make appropriate judgments, it is important to obtain information that accurately indicates patients' levels of comprehension, cognitive function, physical and psychological status, and living situations. Therefore, patients' decision-making capacity needs to be assessed repeatedly, not just once.

When dementia is suspected, it is advisable for healthcare teams to consult with families and cooperate with doctors specializing in dementia or with dementia support doctors. If it is difficult to confirm patients' decisions, their prior instruction (written or oral) should be respected. Healthcare teams and families should support patients so that patients can make the best of their capacity and receive the best healthcare management and care that is consistent with their wishes.

Additionally, it is important to inform patients of their right to prepare advance directives at an early stage when their comprehension and cognitive function are normal or only mildly impaired.

2. Encourage patients' families to engage in advance care planning with patients while they still have decision-making capacity

It is advisable for healthcare teams to encourage families of patients with impaired comprehension and cognition to engage in advance care planning with patients at an early stage when patients still have some decision-making capacity. Consequently, patient-centered discussions about future prospects and healthcare management and care at the EOL should be held, and an advance directive should be prepared. 


\section{Reference materials}

\section{Confirmation form for the temporary decision to forgo dialysis}

Date of confirmation: (year)/(month)/(day)

Doctor providing information:

Attendee(s):

$\begin{array}{lll}\text { Name } & \text { (occupation } \\ \text { Name } & \text { (occupation }\end{array}$

口 1) I received information from my doctor in charge (name: ) and the healthcare team that I am in the end stage of kidney function and that my current condition requires starting or continuing renal replacement therapy (kidney transplantation, peritoneal dialysis, or hemodialysis). I was able to ask the healthcare team questions, and I understand the content of the information.

$\square$ 2) I received information stating that by not starting or not continuing dialysis, I can avoid the pain and time constraints associated with dialysis therapy but that the following symptoms and medical conditions will occur in the near future and that even though I will receive treatment to relieve pain, I will die. I was able to ask questions, and I understand the information provided.

$\square$ (1) Systemic edema and/or serious dyspnea

$\square$ (2) Loss of appetite, nausea, vomiting, and/or diarrhea

$\square$ (3) Anemia and/or bleeding

$\square$ (4) Numbness, sensory disturbance, and/or general malaise

$\square(5)$ Itchiness

$\square$ (6) Convulsions and/or disturbed consciousness

$\square$ 3) I received information stating that I can withdraw my decision at any time, but that depending on the situation at the time of withdrawing my decision, it may not be possible to start dialysis quickly and safely and that I may die because of that. I was able to ask questions, and I understand the information provided. $\square 4)$ I received and understood all the above information, and I wish to opt for conservative kidney management instead of starting or continuing dialysis.

(year) (month) (day)

Patient's signature:

Or proxy's signature: (relationship to the patient: )

Signature of family member, etc.: (relationship to the patient: )

Patient's name ( )

Submitted to: (hospital/clinic)

Doctor's name

\section{Confirmation form for ending the temporary decision to forgo dialysis}

$\square$ I wish to change my decision, and

$\square$ I wish to opt to start or continue temporary hemodialysis

$\square$ I wish to opt to start or continue continuous renal replacement therapy

$\square$ I wish to opt for hemodialysis

$\square$ I wish to opt for peritoneal dialysis

$\square$ I wish to opt for kidney transplantation

(year) (month) (day)

Patient's signature:

Or proxy's signature: (relationship to the patient: )

Patient's name (

Submitted to: (hospital/clinic) Doctor's name 


\section{Footnotes \\ ${ }^{*}$ Healthcare team}

A healthcare team comprises a physician in charge, nurses, and clinical engineers. If possible, other healthcare professionals (e.g., social workers, dieticians, registered dieticians, and pharmacists) and long-term care professionals (e.g., care workers and care managers) should be added to form healthcare management and care teams. Before shared decision making on the temporary decision to forgo dialysis is performed, it is advisable for more than two physicians to judge whether the patient is at the EOL. If the situation is too urgent to form such a team, a physician should make a medically valid and relevant judgment according to the principle that the patient's life should be respected. Then, the subsequent appropriate healthcare can be discussed in the healthcare team. Healthcare teams may perceive difficulties in the decision-making process about the initiation and continuation of dialysis, so it is advisable to form a team or a division responsible for handling clinical ethics-related issues within healthcare facilities.

\section{${ }^{* 2}$ Advance directive}

An advance directive is a written document describing the decisions about details of future healthcare management and care made by individuals irrespective of the presence of disease. With an advance directive, an individual's own decision making, that is, his or her personal autonomy, is respected even when he or she becomes incapable of making decisions. A living will is a written document that gives instructions to the healthcare team regarding the content of healthcare management and care in case something happens to the individual. However, a living will can be vague in some ways because stating concrete and precise instructions for an unknown future is difficult. A durable power of attorney authorizes a surrogate decision maker, and it remains in effect when patients become unable to make decisions. When there are no family members or other relevant persons or when it is difficult to select key persons among many family members, a durable power of attorney indicates whom should be consulted, although preparing a durable power of attorney is not mandatory in Japan. It is also questionable whether the decisions made by surrogates are truly consistent with patients' decisions.

\section{${ }^{*}$ Families and other relevant persons}

The Commentary on the Guidelines for the DecisionMaking Process in End-of-Life Healthcare Management and Care by the MHLW considers future increases in the number of single-person households and states that the family of a patient can comprise more than two individuals whom the patient trusts and who will support the patient at the EOL. In other words, family is not limited to the legally recognized family but includes wider relations (e.g., close friends). Overseas, family includes healthcare proxies (preappointed individuals who make decisions on behalf of the patient based on the prediction of decisions that the patient would have made).

The number of individuals without sufficient decisionmaking capacity who live alone with no close relatives is increasing in Japan's aging society, which also has a low birthrate. In 2019, to address this situation, the MHLW published the Guidelines Regarding Support for Individuals Who Have Difficulties in Decision Making on Matters about Hospitalization and Healthcare to help those who have no relatives and those who have families but whose family members are not approachable or supportive. In the guidelines, individuals without close relatives are classified into three groups (those with adequate decision-making capacity, those without adequate decision-making capacity and under adult guardianship, and those without adequate decision-making capacity and not under adult guardianship), and detailed procedures that healthcare facilities should follow for each of the three groups are provided. The guidelines indicate measures that healthcare facilities should take to provide appropriate healthcare to patients, even those without adequate decision-making capacity, and they summarize specific roles that guardians are expected to play.

If patients have family members, it is important to have discussions with heirs. In Japan, if patients have no family members, adult guardians and voluntary guardians currently do not have the authority to provide consent to healthcare practice, although there may be new legislation or amendment of existing laws in the future. Additionally, if the current adult guardianship system is amended to allow doctors to become adult guardians and/or supervisors of adult guardians, such doctors may be able to express opinions close to consent to the healthcare team. Because doctors currently do not have the authority to provide consent to healthcare or care for patients, it is advisable to check whether patients are public assistance recipients, users of the system supporting independent daily life, users of a guarantor organization, and/or users of long-term care and welfare services for individuals with disabilities and to consult with longterm care professionals (e.g., care workers and care managers) and regional welfare officers.

\section{${ }^{*}$ Heirs}

There are presumptive heirs and lawful heirs. Presumptive heirs are those who would be entitled to inheritance in the future: the primary presumptive heirs are the spouse and children of the deceased person, the secondary heirs are the spouse and parents of the deceased 
person, and the tertiary heirs are the spouse and brothers and sisters of the deceased person. When the individual dies, his or her true successors are determined in accordance with the law. The reason why heirs can be presumed but not determined before the individual's death is that presumptive heirs may die before the individual dies. Nevertheless, unless there are causes for disinheritance or disqualification, presumptive heirs usually become lawful heirs.

If presumptive heirs live with or have a close relationship with the patient, they are also categorized as family, and thus, there is no need to recognize them as presumptive heirs. However, if there are relatives who live separately or do not have a close relationship with the patient, then it is possible that some of them would be presumptive heirs. Such presumptive heirs, irrespective of how estranged they are from the patient, are the persons who are most likely to be able to singlehandedly exercise legal rights (e.g., claim damages and loss on behalf of the patient and claim compensation). Thus, from a legal perspective, if healthcare teams obtain consent to healthcare from every presumptive heir, the problem of possible reparations in the future can be almost completely avoided.

If patients' relatives live independently or are estranged, it is not easy to identify presumptive heirs without examining the family registry if patients' relatives live independently or are estranged. Additionally, some presumptive heirs may be confused by suddenly being asked to provide consent and thus may not respond positively. Therefore, obtaining consent from all presumptive heirs is valuable, albeit not very practical, for healthcare teams to do.

Guardians may be able to reveal presumptive heirs. Guardians sometimes seek opinions from the presumptive heirs of the ward before the management and deposition of important property, and they therefore know the persons who would be beneficiaries. Guardians are not expected to provide consent to healthcare teams under the current system, but they may be able to provide information about presumptive heirs, so there is some value in asking guardians to provide information about presumptive heirs of the ward.

\section{"5 The "Miawase" approach to dialysis}

A temporary decision to forgo dialysis that allows for the later initiation or reinitiation of dialysis depending on changes in the patient's decision or disease status. This temporary decision to forgo dialysis is widely known as "Miawase" in Japanese, and notably, the "Miawase" approach is different from permanently forgoing (withdrawing or withholding) and discontinuing dialysis. In this proposal, we refer to this "Miawase" approach in Japan as the "temporary decision to forgo dialysis".

\section{${ }^{* 6}$ Grief care}

Grief care is supportive care provided during the process through which people overcome grief after the death of loved ones and accept the pain and environmental changes associated with separation by death. According to the Japan Grief Care Association, grief care is gentle support with empathy for people experiencing grief after the death of loved ones when they cope with emotional swings between "overwhelming feelings and emotions toward (mostly attachment to) the deceased" and "the will to overcome such tough circumstances" and thus become very unstable and experience physical discomfort and responses.

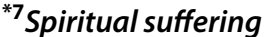

According to the WHO's definition of cancer pain relief and palliative care, "spiritual" is an empirical aspect of human life and describes experiences that transcend physical sensory phenomena. For many people, the spiritual aspect of living includes a religious factor, but "spiritual" is not the same as "religious". The spiritual aspect is a constituent of one's entire life in which physical, psychological, and social factors are involved, and it is often associated with interest in and concerns about the purpose of living. For those who are approaching the final moments of life, spirituality is often associated with selfforgiveness, reconciliation with others, and confirmation of personal values, among other elements. It is difficult to translate spiritual suffering into Japanese: it is sometimes translated as "spiritual pain/distress/agony", but spiritual suffering can be more existential. Spiritual suffering includes questions about meaning and motivation in life, loneliness, unrest, despair, changes in one's value system, the meaning of pain, a sense of guilt, fear of death, the pursuit of God, and anxieties related to views of life and death.

\section{${ }^{* 8}$ Regional welfare officers}

Regional welfare officers have the authority to bury deceased persons who are registered in the family registry but have no relatives (Graveyards and Burials Act), those who are not identifiable and are not registered in the family registry (Act on Treatment of Persons Who Contracted Disease or Died on Journey), or those with relatives but who refused contact with them prior to their death (Public Assistance Act). However, regional welfare officers do not have the legal authority to act as surrogate decision makers in healthcare.

\section{${ }^{* 9}$ Consultation with the head of the facility}

When consensus is not reached among the patient, family, and healthcare team, the team will consult with the head of the facility to hold a nonregular committee 
comprising multiple specialists including a doctor, nurses, clinical engineers, and a healthcare ethics specialist or a standing ethics committee. This committee advises the team, which aims to reach consensus with patients and their families. Depending on the situation, a conference by medical stuff and long-term care workers other than the doctor, nurse, and clinical engineer in charge can be utilized.

\section{Concluding remarks}

The JSDT formulated this proposal so that medical teams can provide patients with the best healthcare management and care at the time of decision making on the initiation and continuation of dialysis. Unlike guidelines for diagnosis and medical care for diseases, this proposal was formulated to reflect individual patients' medical conditions and life perspectives, their families' thoughts, and other such considerations. Furthermore, this proposal is based on the results of observational studies and the current consensus in Japan and overseas rather than on objective results (i.e., evidence) that can be adapted to many situations.

This proposal follows the motto of the Japan Association of Kidney Disease Patients established in 1971"Safe dialysis for anybody, anytime, and anywhere"-and presents a decision-making process that can be used in difficult cases that are often encountered by healthcare teams in daily clinical practice. In this way, patients can receive dialysis therapy at any dialysis facility without worry. Notably, this document is a consensus-based proposal, not evidence-based clinical practice guidelines, and it is not based on legal interpretations of related laws.

Before dialysis must be initiated, healthcare teams should consider how to provide information about CKM that includes nondialysis medical management and care for ESKD in the following situations: when patients submit advance directives to healthcare teams; when patients and their families request the Japanese "Miawase" approach to dialysis (the temporary decision to forgo dialysis); and when healthcare teams judge that patients are in a condition that makes this approach is an option (Table 1). When the initiation of dialysis is necessary, healthcare teams should provide information about CKM when patients do not opt for RRT and when the temporary decision to forgo dialysis is requested by patients and their families. After providing such information, healthcare teams should engage in the decisionmaking process with patients and their families so that decisions are made through shared decision making and advance care. If CKM is requested, it is important that healthcare teams understand patients' personal values and intentions, tell the patients and their families that the patient's satisfaction in life is most important, aim for consensus building through discussions, and thereby support patients in making the best decisions for them.

If CKM, in addition to RRT, is included in the medical care options for ESKD, four medical care options (kidney transplantation, peritoneal dialysis, hemodialysis, and CKM) should be provided, as is done overseas. Patients have the right to know their options, and healthcare teams should provide them with all necessary information. Healthcare teams are expected to comprehensively judge patients' disease status and comprehension levels and appropriately provide information about the timing, procedure, intensity, and details of medical care options.

It is necessary to establish the content and practice of care for patients who opt for the temporary decision to forgo dialysis after choosing CKM in collaboration with home-cased care doctors, with differences between Japan and Europe/North America taken into account.

It is important for healthcare teams to respect patients' decisions, understand their intentions, and support them in living their lives with dignity and without regret and in living their final moments as they wish. Healthcare teams also need to provide patients' families with psychological care (e.g., grief care), allowing them to feel satisfied that the patients lived their final moments as they wished.

The JSDT hopes that this proposal helps promote awareness and discussion of this common issue, particularly about how to live at the EOL, among all Japanese citizens and hopes that better healthcare management and care will be provided as a result of such discussion.

\section{Basic concepts in the development and commentary on the proposal Respecting patients' decisions}

The MHLW stated that healthcare management and care at EOL should be provided in such a way that individuals can live their own lives until their final moments with their dignity respected and upheld [3]. To achieve this goal, patients' decisions, or inferred decisions if they are not capable of making decisions, should be respected.

The Japan Geriatrics Society stated its position on EOL healthcare management and care for aged individuals in 2012 based on the notion that, when reaching the final moments of life, all individuals have the right to receive the best possible healthcare management and care in a manner that respects their personal values, thoughts, beliefs, and religion [26]. More precisely, irrespective of the required care level or the presence of dementia, older individuals have the right to receive the best possible healthcare management and care for them, and for those at the EOL, respect should be paid to their values, thoughts, beliefs, and religion in a manner consistent with the sense of family and ethics unique to Japan. Based on this position statement, the Japan Geriatrics Society 
published Guidelines for the Decision-making Processes in Medical and Long-Term Care for the Elderly: Focusing on the Use of Artificial Hydration and Nutrition in 2012 [27].

Currently, more patients on dialysis have impaired comprehension or cognition than in the past, but they still hold the decision-making authority. Thus, because these patients have decision-making capacity, it is important to apply the principle of consent with them and to support them in making decisions in accordance with the Guidelines for Supporting Decision Making in Daily and Social Life for Individuals with Dementia [7] published by the MHLW. It is particularly important that patients and their families hold patient-centered discussions about the healthcare management and care that will be necessary for them in the future while patients' comprehension and cognition are still normal or while impairment is still at an early stage.

What patients say (emotions) should not be taken lightly when inferring their true feelings (meanings). The principal attitudes of healthcare teams should support the identification and understanding of patients' personal values through communication and to use that information during discussions with them.

\section{Principal attitudes of doctors and healthcare teams}

In compliance with the Medical Care Act of Japan, doctors understand the physical and mental states of patients and provide appropriate healthcare accordingly. As specified in the Japan Medical Association's Guidelines for Physicians' Professional Ethics [28], doctors prioritize acting in patients' interests and respect and protect their rights, such as their right to receive fair treatment and their right to decline treatment. Furthermore, the Code of Medical Ethics states that those in medicine and healthcare should not only treat patients but also help people maintain or enhance their health: doctors should recognize their significant responsibilities and compassionately serve everyone.

In compliance with the Japanese Nursing Association's Code of Ethics for Nurses, nurses should respect patients' decision making, ensure that patients receive the necessary information and decision-making opportunities, and support them with a warm sense of humanity.

Other professionals in healthcare teams work with doctors and nurses while respecting patients' decisions, and in this way, they provide good healthcare as a team so that patients can live their lives and their final moments as they wish.

\section{Medical care options for ESKD}

A pamphlet made by five societies (Treatment Options for Kidney Failure and the Current Status, 2020 Edition) [29] provides information about kidney transplantation, peritoneal dialysis, and hemodialysis as medical care options for ESKD but does not provide information about CKM, which is nondialysis medical care and care $[8,30]$. ESKD is generally considered a condition in which symptoms of uremia develop due to the failure of renal compensatory mechanisms. The Japanese Society of Nephrology defines ESKD as stage 5 CKD [31].

Information about RRT should be provided to patients with progressive deterioration of kidney function when the eGFR becomes less than $30 \mathrm{~mL} / \mathrm{min} / 1.73 \mathrm{~m}^{2}$. Healthcare teams must provide patients with information about alternatives as well as the proposed medical care. When RRT is considered a realistic option in the near future, healthcare teams should provide patients with information on RRT and the natural course of ESKD, and based on this information, some patients will opt for RRT before the onset of symptoms of uremia, while others will not. However, many patients who do not opt for RRT at this point will later change their decisions and accept RRT after experiencing severe symptoms of uremia. Such changes are more common in medical care for ESKD than for other diseases, indicating that the timing of the provision of information about CKM should be judged carefully.

Patients who have not yet opted for RRT are faced with a decision-making situation when they reach the stage of ESKD when the initiation of dialysis becomes necessary. Patients should be provided with information about the benefits and risks of undergoing and not undergoing RRT, the different types of RRT modalities (kidney transplantation, peritoneal dialysis, and hemodialysis), and the natural course of the disease if RRT is not initiated. If patients request a non-RRT option, then healthcare teams should provide information about CKM.

\section{Shared decision making and second opinions in the "Miawase" approach to dialysis}

A temporary decision to forgo dialysis allows for the later initiation or reinitiation of dialysis depending on changes in the patient's decision or disease status. This temporary decision is widely known as "Miawase" in Japanese, and notably, the "Miawase" approach is different from permanently forging (withdrawing or withholding) and discontinuing dialysis.

When patients, together with their families, decide to opt for CKM, healthcare teams should participate in the shared decision-making process with them and help them decide on medical care and care after the temporary decision to forgo dialysis is determined. There are many options, and choosing the best option for the patient is not easy. Healthcare teams should provide patients with evidence-based medical information in a clear and understandable way; identify patients' personal 
values, intentions, and concerns; make proposals based on medical knowledge and experience; and have discussions with patients to ascertain their thoughts. Patients' understanding of the information given should be assessed regularly, and it is ideal for patients to determine the best option for themselves through this process [32]. If patients do not change their decisions, a form confirming the temporary decision to forgo dialysis should be obtained as necessary. If patients make regular outpatient visits, healthcare teams should confirm whether their decisions have changed, examine their disease status, and provide palliative care information.

In decision making on CKM, healthcare teams should show empathy toward patients, establish a relationship of trust with patients as much as possible, participate in the shared decision-making process, and support patients in choosing the best possible option for them. If patients are unsure about their decisions, they should be provided with information about the availability of second opinions by specialists at other medical facilities. Even after patients decide on the healthcare management and care options available to them, their conditions should be carefully followed up, and it should be assessed whether the options that have been chosen have been well implemented and whether they remain the best options for the patients. Opportunities to review decisions should be provided if necessary.

When patients are incapable of making decisions, healthcare teams should hold discussions with the patients' families or other relevant persons to identify proxies. When there are no family members or other relevant persons or when patients can give decision-making authority to the healthcare team, the healthcare team should consult with care providers (e.g., care workers and long-term care managers) or regional welfare officers to provide the patient with the best healthcare management and care in accordance with his or her remaining abilities. If care providers and regional welfare officers are not available for consultation, the healthcare team should consult with the head of the corresponding facility.

\section{Shared decision making, advance directives, and advance care planning}

Shared decision making is a process of communication in which healthcare teams and patients work together to make optimal healthcare decisions for patients [33]. Important aspects of this process are (1) evidence-based medical information; (2) proposals from healthcare teams; and (3) sharing of patients' personal values, preferences, concerns, etc., among all parties.

An advance directive is a document that describes patients' decisions about healthcare management and care in advance so that the autonomy of patients is respected even after they have become incapable of making decisions for themselves. An advance directive can be made after repeated discussions among the patient, the family, and the healthcare team or by the patient himself or herself; nevertheless, the advance care directive should be reviewed from time to time because decisions can change.

A consensus conference to establish a unified conceptualization of advance care planning was held in 2017, and a consensus definition of advance care planning was developed as follows (although it will evolve over time): "Advance care planning is a process that supports adults at any age or stage of health in understanding and sharing their personal values, life goals, and preferences regarding future medical care. The goal of advance care planning is to help ensure that people receive medical care that is consistent with their values, goals and preferences during serious and chronic illness" [34].

In Japan, the concept of advance care planning first appeared in 2018 in the revised Guidelines for the Decision-Making Process in End-of-Life Healthcare Management and Care [3]. It has been actively promoted using its nickname "life meeting" (Jinsei kaigi in Japanese) [35]. In 2019, the Japan Geriatrics Society defined advance care planning as "a process that supports patient's decision-making regarding future healthcare management and care, during which patients are respected as human beings" to ensure that patients receive EOL healthcare management and care that aligns with their intentions, thereby allowing them to live fulfilling lives until their final moments, with their dignity respected and upheld [36].

Advance care planning is more than a sequence of steps taken for EOL healthcare (e.g., the preparation of advance directives and the appointment of proxies). In the process of advance care planning, an advance directive is prepared, proxies are appointed, and decisions regarding the initiation and discontinuation of certain healthcare interventions are made. In other words, advance care planning facilitates the process of shared decision making that aligns healthcare management and care with individuals' personal values, goals, and preferences [37]. The content of an advance directive should be reviewed and updated as necessary.

\section{Proxies}

When patients cannot express their decisions or lack decision-making capacity, those who can infer the patients' decisions about healthcare management and care (e.g., family members) become proxies, but it is important to involve legal heirs in discussion. If there are no family members, the Guidelines Regarding Support for Individuals Who Have Difficulties in Decision Making 
on Matters about Hospitalization and Healthcare, published by the MHLW in 2019, should be referred to [38]. However, guardians of adults and voluntary guardians do not have the authority to consent to patients' healthcare management and care; thus, it is preferable that healthcare teams consult with known long-term care professionals and welfare officials. If contactable legal heirs are identified after the family registry is examined, their consent is legally significant.

When consensus is not reached with patients' families or when patients' families are not cooperative or cannot be contacted, healthcare teams should consult with the heads of their facilities.

\section{EOL}

In the Commentary on the Guidelines for the DecisionMaking Process in Terminal-Stage Healthcare [39] published by the MHLW, patients are considered to be in the terminal stage when the predicted survival is a few days or a few weeks at the longest, as in the end stage of cancer; when poor prognosis is expected after repeated acute exacerbation of a chronic condition; or when the predicted survival is a few months to a few years in patients with sequelae of cerebrovascular disease or individuals with poor general conditions. Healthcare teams must appropriately identify the terminal stage for each patient based on the individual situation. The Japan Medical Association's Grand Design 2007 (Particulars) distinguishes terminal-stage care in a broad sense from terminal-stage care in a narrow sense. It defines the terminal stage in a broad sense as follows: (1) the terminal stage is the stage in which progressive exacerbation cannot be stopped and patients are reaching the final moments of their lives, even though the best possible healthcare is provided; (2) the terminal stage starts when the condition described in (1) is confirmed by doctors, including the doctor in charge, nurses, and several other healthcare professionals, and is understood and agreed upon by patients (or families who can infer patients' decisions if patients are incapable). The terminal stage in the narrow sense is defined as the stage when patients are dying and very close to the final moments of their lives.

The present proposal uses "EOL" rather than "terminal stage" in accordance with recent guidelines published by the MHLW. The actual condition corresponding to the EOL is determined for individual patients by doctors based on an appropriate assessment of the patient's systemic conditions (including dialysis-related complications and other illnesses). EOL starts when the patient (or the family if the patient does not have decision-making capacity) understands and provides consent to doctors' judgments. In ESKD, the necessity of dialysis alone does not determine EOL. However, if a patient who is not at the EOL stage medically but requires life-sustaining dialysis opts for the temporary decision to forgo dialysis in CKM, it is highly likely that he or she will survive only a few days or weeks. The criteria for the initiation of maintenance dialysis stated in the MHLW Project Report on Healthcare for Renal Failure published in 1991 [40] have been used for the initiation of dialysis. However, cases of earlier initiation have been on the rise since the Guidelines for Peritoneal Dialysis [41] and the Guidelines for Maintenance Hemodialysis [42] were published by the JSDT in 2009 and 2013, respectively; these guidelines established revised criteria for the initiation of peritoneal dialysis and maintenance hemodialysis. Additionally, dialysis may become unnecessary in some patients with acute exacerbation of CKD or acute kidney injury. Overall, if the temporary decision to forgo dialysis is requested by patients with decision-making capacity or by the families of patients without decision-making capacity, patients can be regarded as being at the EOL stage.

The presence of severe cognitive impairment alone is not a criterion for EOL. Additionally, the frequency of the temporary decision to forgo dialysis was found to be similar between patients with severe cognitive impairment and those in the terminal stage of malignancy [43, 44]. Thus, when making decisions about healthcare management and care strategies for patients with dementia who are not at the EOL and who do not have decisionmaking capacity, healthcare teams should confirm any prior instruction provided by the patient (written or oral) together with the family and judge whether the temporary decision to forgo dialysis proposed by the family is a surrogate decision and not a decision based on the wishes of the family.

\section{Assessment of decision-making capacity}

The assessment of a patient's decision-making capacity involves the patient, his or her family, and the healthcare team. When assessment is not straightforward, sufficient time should be spent to comprehensively examine the following capacities: understanding of issues necessary for decision making; reasoning in decision making (comparison between options based on the patient's personal values); appreciation of illness, medical care, and decision making as the patient's own affairs; and communication of the patient's own thoughts and conclusions.

\section{The temporary decision to forgo dialysis Proposal of the temporary decision to forgo dialysis by the healthcare team}

Healthcare teams can propose the temporary decision to forgo dialysis to patients with decision-making capacity or to the families of patients without such capacity when patients satisfy certain conditions (see Table 1). When 
patients at the EOL stage and/or their families choose CKM in accordance with the decision-making process (Fig. 1) and when the patients and their families consent to the temporary decision to forgo dialysis, this approach can be implemented. A confirmation form for the approach should be obtained from patients and/or their families (including heirs) if necessary. When patients without decision-making capacity are not at the EOL stage, the procedure described in 9.3 below is applied.

\section{Request for the temporary decision to forgo dialysis by patients with decision-making capacity}

Healthcare teams should verify temporary decisions to forgo dialysis and the reasons for these decisions. It is not uncommon for patients with no prior experience of dialysis to decline dialysis therapy initially but to eventually accept dialysis after making regular visits to healthcare facilities. Additionally, patients who have been on dialysis may propose the temporary decision to forgo dialysis and later reverse this decision to reinitiate dialysis. When doctors judge that patients are at the EOL stage, information should be provided about the possibility of a stepwise reduction in the duration and frequency of dialysis, and if consent is obtained from patients and their families, the temporary decision to forgo dialysis can be implemented. Any change in the temporary decision regarding this approach should be confirmed while providing appropriate palliative care based on routine examinations. A confirmation form for the temporary decision to forgo dialysis should be obtained from patients and/or their families (including heirs) if deemed necessary.

Patients with decision-making capacity, irrespective of whether they are at the EOL, have the right to receive clear and appropriate information and the right to make their own decisions to accept or decline healthcare interventions. For patients who are not yet in the EOL stage who request the temporary decision to forgo dialysis and eventually opt for CKM, EOL starts when a doctor diagnoses ESKD that requires maintenance life-sustaining dialysis. For patients not at the EOL who opt for the temporary decision to forgo dialysis, their decision should be regarded as final at that point and should be respected. However, healthcare teams must always be aware that patients may change their minds, and they should be prepared to initiate dialysis for them. Autonomy-that is, making one's own decisions about one's own affairs-is the most important element of human dignity, and therefore, consensus achieved among the patient, family, and healthcare team to opt for CKM, after thorough discussion based on appropriately shared essential, should be respected. A confirmation form for the temporary decision to forgo dialysis should be obtained from the patient or their families (including heirs) if necessary. If consensus is not reached among the above parties, consensus-building discussion should be continued.

\section{Request for the temporary decision to forgo dialysis by families of patients without decision-making capacity}

Families of patients without decision-making capacity have no choice but to become surrogate decision makers regarding the patient's healthcare management and care. When the temporary decision to forgo dialysis is requested by families, healthcare teams should confirm with the families whether there are any prior instruction (written or oral) from the patient and the reasons for any decisions made by the patient, and they should ensure that the requests are based on surrogate decisions, not on the wishes of the family. When consensus for CKM is achieved with families after sufficient information sharing and adequate discussion, then healthcare teams should recognize that the families' decision making protects patient dignity, and thus, the decision reached based on consensus should be respected. Once the above is confirmed, the request for the temporary decision to forgo dialysis made by families and the requests made by patients themselves should be handled in a similar manner: a confirmation form for the temporary decision to forgo dialysis should be obtained if deemed necessary. If patients' decisions cannot be inferred or if consensus is not reached with families, consensus-building discussions should be continued.

\section{Provision of information to patients and their families or other relevant persons}

Providing information about the disease status and other aspects to patients and their families or other relevant persons is a doctor's responsibility. Doctors are required to provide appropriate information about the timing, procedures, intensity, and content of healthcare management and care based on a comprehensive assessment of the disease status and patient comprehension.

When information about the disease status is clearly and appropriately provided to patients with decisionmaking capacity and patients' decisions are respected, doctors are not necessarily required to provide information to patients' families. Information should be provided to families if patients do not have decisionmaking capacity. Nevertheless, given that families are most likely the people who support patients, to avoid future conflicts with them, it is advisable to keep them informed if patients approve. When patients' families who closely support patients act as proxies, instead of asking about the families' decisions and wishes, questions such as the following should be posed: "What would the patient say if he or she could communicate his or her decision?". 
In some cases, patients refuse to involve their families to avoid troubling them, or family members are not appointed as decision-making supporters. In such cases, the reasons for the lack of family involvement should be ascertained.

When uremic symptoms appear or when CMK is chosen and the temporary decision to forgo dialysis is implemented, patients may have their final moments earlier than their families expected. It is beneficial for patients reaching their final moments at the EOL to obtain the support and attention they expect from their families after their families have received adequate information; the provision of this information to families deserves legal protection. Thus, it is advisable to provide information about the disease status to families after informing patients about their disease status.

\section{Palliative care and EOL care}

Palliative care involves medical, nursing, and long-term care to alleviate and prevent symptoms of physical, emotional, and spiritual suffering during the entire clinical course, from diagnosis to EOL; such care can be provided on an outpatient or inpatient basis at integrated facilities for medical and long-term care, at long-term care facilities, or at home, according to wishes of patients and their families [45].

Palliative care should be provided even before a temporary decision to forgo dialysis is made. If this approach and home-based care are chosen when the patient is reaching the final moments of life, healthcare teams should provide the doctors responsible for home-based care with information about the clinical course, test results, the decision-making process, advance care planning details, key persons, and the presence/absence of dementia (history of medical care); health care teams should also provide doctors with a copy of the confirmation form for the temporary decision to forgo dialysis [46]. Healthcare teams remain responsible for providing palliative care at patients' homes if the involvement of home-based care doctors is declined by patients or their families. Healthcare teams should provide patients and their families with detailed information about palliative care as well as healthcare management and care for predicted symptoms (e.g., general malaise, pruritus, swelling, loss of appetite, nausea, vomiting, dyspnea, and disturbance of consciousness).

According to the MHLW guidelines, the provision of care to alleviate physical suffering as early as possible is important in healthcare management and care in the EOL stage. Given that dyspnea and other symptoms due to uremia cause intolerable suffering, it is important to confirm patients' decisions about palliative care.
Additionally, for continuous deep sedation, it is advisable to obtain prior consent from patients and their families.

EOL care should assist individuals facing imminent death or thinking about their death in the future to live their lives to the fullest, regardless of their diagnosis, health condition, and age [47]. To provide the necessary EOL care, healthcare teams should discuss healthcare management and care that will become necessary in the future with patients and their families while patients are still healthy or at an early stage of illness.

\section{Children}

It is advisable to decide whether CKM is in the best interest of children through the exchange of various opinions among relevant people with different perspectives (e.g., the healthcare team and the patient's family members) in compliance with the Guidelines for Communication of Medical Policy on Children Suffering from Serious Diseases published in 2012 by the Japan Pediatric Society [48].

\section{Documentation of discussions}

All professionals in healthcare teams should record all remarks made by themselves, patients, and patients' families during any discussions and should share written summaries with patients and their families.

\section{Consent form and confirmation form}

Providing "consent" means agreeing to others' thoughts and opinions or expressing decisions that are the same as others' decisions: an individual gives consent when he or she accepts another's opinion. Meanwhile, "consensus" is mutual agreement, and the people involved have equal positions in terms of the acceptance of their individual standpoints; in the context of shared decision making, consensus is achieved when all parties have expressed their own opinions and have reached an agreement or accept the conclusion of the discussion. When patients decide to initiate dialysis or their families provide consent to the initiation of dialysis based on adequate information on the treatment provided by healthcare teams, a consent form should be completed for confirmation. On the other hand, when patients opt for the temporary decision to forgo dialysis but healthcare teams consider the initiation of dialysis to be clearly beneficial to them, the patients' decisions still need to be respected, and a confirmation form should be completed if the teams can agree on why the patients and their families made their decisions.

The content of discussions with patients and their families held before CKM is selected should be recorded in medical documents, but notably, medical documents cannot serve as proof of consent from patients and their 
families. Having both a consent form and a confirmation form provides solid evidence of the decision, the provision of consent, and the achievement of consensus among the relevant parties in case any future conflict arises. A consent form for the initiation of dialysis must be obtained, and a confirmation form for the temporary decision to forgo dialysis should be obtained if necessary.

\section{Establishment of a team or a division responsible for issues related to clinical ethics}

Healthcare teams may have difficulties in the course of decision making about the initiation and continuation of dialysis. It is advisable for a team or a division responsible for handling issues related to clinical ethics to be established in individual healthcare facilities.

\section{Principles for formulating the proposal Objectives}

The aim of this proposal is for healthcare teams to participate in decision-making processes in which patients' decisions are respected and their intentions are understood so that patients can live their lives with their dignity respected and upheld and can live their final moments as they prefer.

\section{Target users}

This proposal is for doctors, other healthcare professionals (e.g., nurses, clinical engineers, social workers, dietitians, registered dietitians, and pharmacists), and long-term care professionals (e.g., certified care workers and long-term care managers) who are involved in the healthcare management and care of patients with CKD and patients on dialysis.

\section{Target patients}

Patients with CKD (from pediatric to elderly patients) and patients on dialysis.

\section{Clinical problems addressed}

Even when the temporary decision to forgo dialysis is decided with full respect for the patient's decision and implemented in compliance with this proposal, there is no legislation in place regarding the withdrawal of lifesustaining medical care in Japan, and healthcare teams are not exempt from legal liability for consequent deaths.

\section{Ensuring universality}

To avoid bias based on the opinions of the JSDT, the following societies were asked to contribute to the selection of committee members: the Japanese Society for Peritoneal Dialysis, Japanese Association of Dialysis Physicians, Japanese Society of Nephrology, Japanese Society for Pediatric Nephrology, Japan Academy of Nephrology
Nursing, Japan Association for Clinical Engineers, Japanese Association for Home Care Medicine, and Japan Shared Decision Making Collaborative for Chronic Kidney Disease. Additionally, as representatives of patients, the Japan Association of Kidney Disease Patients and the Kidney Support Association were asked to contribute.

\section{Format}

Given the limited evidence available in this field, the format of clinical questions was not used. Instead, the previous proposals and the MHLW's Guidelines for the Decision-Making Process in End-of-Life Healthcare Management and Care were used as reference materials. When agreement among all committee members was not reached, the issues were discussed and decided at the JSDT board meeting.

\section{Literature search}

The literature published in English and Japanese between April 2012 and June 2019 was searched in the PubMed and Ichushi databases. Handsearching was performed for crucial studies. The keywords used included shared decision making, hemodialysis, peritoneal dialysis, acute kidney injury, advance directives, death with dignity, selfdetermination, terminal stage, advance care planning, EOL care, palliative care, forgo, withhold, withdrawal, elderly, cognitive impairment, dementia, and CKM or conservative kidney care. Studies providing a higher level of evidence were selected, and studies providing a lower level of evidence or reviews were evaluated at the general meeting to avoid bias.

\section{Abbreviations \\ CKD: Chronic kidney disease; CKM: Conservative kidney management; eGFR: Estimated glomerular filtration rate; EOL: End of life; ESKD: End stage kidney disease; JSDT: Japanese Society for Dialysis Therapy; QOL: Quality of life; RRT: Renal replacement therapy.}

\section{Supplementary Information}

The online version contains supplementary material available at https://doi. org/10.1186/s41100-021-00365-5.

Additional file 1. Supplementary material on the principles for formulating the proposal.

\section{Acknowledgements}

This paper has been completed with the efforts of the external reviewers. We would like to express our deepest gratitude to all these individuals. The Japanese version of this proposal [49] has been published previously the present paper is a duplicated publication with permission from the JDST. Comments on this paper were provided by the reviewers, and we made major revisions to the original work. In publishing this English translation, we hope to share perspectives and practices from Japan and to contribute to shared decision making involving dialysis patients, families, and healthcare teams around the world. Committee for Updating the Proposal for Shared Decision Making on the Initiation and Continuation of Dialysis: Kazuyoshi Okada, Ken Tsuchiya, 
Ken Sakai, Takahiro Kuragano, Akiko Uchida, Kazuhiko Tsuruya, Tadashi Tomo, Chieko Hamada, Masafumi Fukagawa, Yoshindo Kawaguchi, Yuzo Watanabe, Kaoruko Aita, Yoshitatsu Ogawa, Junji Uchino, Hirokazu Okada, Yutaka Koda, Yasuhiro Komatsu, Hisamitsu Sato, Motoshi Hattori, Toru Baba, Mamiko Matsumura, Hisayuki Miura, Jun Minakuchi, Hidetomo Nakamoto.

\section{Authors' contributions}

$\mathrm{KO}$ was in charge of the Introduction, Basic concepts in the development and commentary on the proposal, Principles for formulating the proposal, Individual proposals, Commentary on the individual proposals, Closing remarks, and Notes sections, and YW was in charge of the background on the Proposal for the Shared Decision-Making Process. Regarding the Initiation and Continuation of Maintenance Hemodialysis 2014, MF was in charge of describing the situation surrounding withholding and withdrawal of dialysis in Japan and overseas. In addition, KT was in charge of the part on decision support for people with dementia, $\mathrm{MH}$ was in charge of the pediatric part, and $\mathrm{YO}$ was in charge of the legal part. KS, MT, and TH were in charge of the literature search; TK was in charge of creating reference materials for the confirmation form for the temporary decision to forgo the dialysis approach to dialysis and the confirmation form for ending the temporary decision to forgo the dialysis approach to dialysis; and YK, AU and HS were in charge of the figure on the decision-making process when renal replacement therapy becomes necessary. All authors read and approved the final manuscript.

\section{Funding}

This proposal did not receive any funding. All costs for the creation of the paper were provided by the JSDT.

\section{Availability of data and materials}

Not applicable.

\section{Declarations}

Ethics approval and consent to participate

Not applicable.

\section{Consent for publication}

Not applicable.

\section{Competing interests}

The authors declare that they have no competing interests.

\section{Author details}

'Department of Nephrology, Kawashima Hospital, 1-39 Kitasakoichiban-cho, Tokushima-shi, Tokushima 770-0011, Japan. 2Department of Blood Purification, Kidney Center, Tokyo Women's Medical University Hospital, Tokyo 162-8666, Japan. ${ }^{3}$ Department of Nephrology, Faculty of Medicine, Toho University, Tokyo 143-8541, Japan. ${ }^{4}$ Department of Nephrology and Dialysis, Faculty of Internal Medicine, School of Medicine, Hyogo College of Medicine, Hyogo 663-8501, Japan. ${ }^{5}$ Department of Nursing, Seirei Sakura Citizen Hospital, Seirei Welfare Service Group, Chiba 285-8765, Japan. ${ }^{6}$ Department of Nephrology, Nara Medical University, Nara 634-8522, Japan. ${ }^{7}$ Clinical Engineering Research Center, Faculty of Medicine, Oita University, Oita 879-5593, Japan. ${ }^{8}$ Juntendo Advanced Research Institute for Health Science, Juntendo University, Tokyo 113-8431, Japan. ${ }^{9}$ Division of Nephrology, Endocrinology and Metabolism, Tokai University School of Medicine, Kanagawa 259-1193, Japan. ${ }^{10}$ The Jikei University School of Medicine, Tokyo 105-0003, Japan. ${ }^{11}$ Kasugai Municipal Hospital, Aichi 86-8510, Japan. ${ }^{12}$ Uehiro Division for Death and Life Studies and Practical Ethics, Graduate School of Humanities and Sociology, University of Tokyo, Tokyo 113-0033, Japan. ${ }^{13}$ Ogawa Sogo Law Office, Tokyo 160-0004, Japan. ${ }^{14}$ Division of ME, Mihama Hospital, Seijinkai, Chiba 261-0013, Japan. ${ }^{15}$ Department of Nephrology, Saitama Medical University, Saitama 350-0495, Japan. ${ }^{16}$ Koda Internal Medicine Clinic, Niigata 959-0264, Japan. ${ }^{17}$ Department of Healthcare Quality and Safety, Gunma University Graduate School of Medicine, Gunma 371-8611, Japan. ${ }^{18}$ Department of Nursing, Masuko Memorial Hospital, Aichi 453-8566, Japan. ${ }^{19}$ Department of Pediatric Nephrology, Kidney Center, Tokyo Women's Medical University Hospital, Tokyo 162-8666, Japan. ${ }^{20}$ The Japan Association of Kidney Disease Patients, Tokyo 113-0021, Japan. ${ }^{21}$ The Kidney Support Association, Tokyo 160-0022, Japan. ${ }^{22}$ Department of Home Care and Regional Liaison
Promotion, National Center for Geriatrics and Gerontology, Aichi 474-8511, Japan. ${ }^{23}$ Department of General Internal Medicine, Saitama Medical University Hospital, Saitama 350-0495, Japan.

Received: 18 February 2021 Accepted: 19 August 2021 Published online: 14 October 2021

\section{References}

1. Ministry of Health, Labour and Welfare. Guidelines for the decision-making process in terminal-stage healthcare. http://www.mhlw.go.jp/shingi/ 2007/05/dl/s0521-11a.pdf. Accessed 12 May 2021.

2. Ministry of Health, Labour and Welfare. Guidelines for the decisionmaking process in end-of-life healthcare. https://www.mhlw.go.jp/file/ 04-Houdouhappyou-10802000-Iseikyoku-Shidouka/0000079906.pdf. Accessed 12 May 2021.

3. Ministry of Health, Labour and Welfare. Guidelines for the decisionmaking process in end-of-life healthcare management and care. https:// www.mhlw.go.jp/file/04-Houdouhappyou-10802000-Iseikyoku-Shido uka/0000197701.pdf. Accessed 12 May 2021.

4. Japanese Society for Dialysis Therapy. Proposal for the shared decisionmaking process regarding initiation and continuation of maintenance hemodialysis. J Jpn Soc Dial Ther. 2014;47:269-85.

5. Okada K, Japanese Society for Dialysis Therapy. Proposal for the shared decision-making process for initiation and continuation of maintenance hemodialysis: survey after introduction. J Jpn Ass Dial Physician. 2019;34:1106.

6. Japanese Society of Neurology. Clinical practice guidelines for dementia 2017. Tokyo: Igaku Shoin; 2017.

7. Ministry of Health, Labour and Welfare. Guidelines for supporting decision making in daily and social life for individuals with dementia. https:// www.mhlw.go.jp/file/06-Seisakujouhou-12300000-Roukenkyoku/00002 12396.pdf. Accessed 12 May 2021.

8. Grove D, McGinty A, Woodhouse J. A guide to conservative kidney management. Oxford: Oxford University Hospital Kidney Unit; 2018.

9. Neu S, Kjellstrand CM. Stopping long-term dialysis. An empirical study of withdrawal of life-supporting treatment. N Engl J Med. 1986;314:14-20.

10. Hirsch DJ, West ML, Cohen AD, Jindal KK. Experience with not offering dialysis to patients with a poor prognosis. Am J Kidney Dis. 1994;23:463-6.

11. Renal Physicians Association and American Society of Nephrology. Shared decision making in the appropriate initiation of and withdrawal from dialysis. Clinical Practice Guideline Number 2. Rockville: Renal Physicians Association; 2000.

12. Renal Physicians Association. Shared decision making in the appropriate initiation of and withdrawal from dialysis. Clinical Practice Guideline. Rockville: Renal Physicians Association; 2010.

13. Ohira S. Refusal, continuation, and discontinuation of dialysis. In: Maeda T, Ohira S, Miki T, editors. Dialysis in the elderly. Tokyo: Nihon Medical Center; 1995. p. 212-21.

14. Saran R, Robinson B, Abbott KC, Agodoa LYC, Bragg-Gresham J, Balkrishnan R, et al. US renal data system 2018 annual data report: epidemiology of kidney disease in the United States. Am J Kidney Dis. 2019;73:A7-8.

15. Aggarwal $Y$, Baharani J. End-of-life decision making: withdrawing from dialysis: a 12-year retrospective single centre experience from the UK. BMJ Support Palliat Care. 2014;4:368-76.

16. Murphy E, Germain MJ, Cairns H, Higginson IJ, Murtagh FE. International variation in classification of dialysis withdrawal: a systematic review. Nephrol Dial Transplant. 2014;29:625-35.

17. Davison SN, Tupala B, Wasylynuk BA, Siu V, Sinnarajah A, Triscott J. Recommendations for the care of patients receiving conservative kidney management: focus on management of CKD and symptoms. Clin J Am Soc Nephrol. 2019;14:626-34.

18. Okamoto I, Tonkin-Crine S, Rayner H, Murtagh FE, Farrington K, Caskey F, et al. Conservative care for ESRD in the United Kingdom: a national survey. Clin J Am Soc Nephrol. 2015;10:120-6.

19. Chan CH, Noble H, Lo SH, Kwan TH, Lee SL, Sze WK. Palliative care for patients with end-stage renal disease: experiences from Hong Kong. Int J Palliat Nurs. 2007;13:310-4. 
20. Lai CF, Tsai HB, Hsu SH, Chiang CK, Huang JW, Huang SJ. Withdrawal from long-term hemodialysis in patients with end-stage renal disease in Taiwan. J Formos Med Assoc. 2013;112:589-99.

21. Morton RL, Webster AC, McGeechan K, Howard K, Murtagh FE, Gray NA, et al. Conservative management and end-of-life care in an Australian cohort with ESRD. Clin J Am Soc Nephrol. 2016;11:2195-203.

22. Verberne WR, Geers AB, Jellema WT, Vincent HH, van Delden JJ, Bos WJ. Comparative survival among older adults with advanced kidney disease managed conservatively versus with dialysis. Clin J Am Soc Nephrol. 2016;11:633-40.

23. Wongrakpanich $S$, Susantitaphong $P$, Isaranuwatchai S, Chenbhanich J, Eiam-Ong S, Jaber BL. Dialysis therapy and conservative management of advanced chronic kidney disease in the elderly: a systematic review. Nephron. 2017;137:178-89.

24. Tsai HB, Chao CT, Chang RE, Hung KY, Cogent Study Group. Conservative management and health-related quality of life in end-stage renal disease: a systematic review. Clin Invest Med. 2017;40:E127-34.

25. Ren Q, Shi Q, Ma T, Wang J, Li Q, Li X. Quality of life, symptoms, and sleep quality of elderly with end-stage renal disease receiving conservative management: a systematic review. Health Qual Life Outcomes. 2019;17:78.

26. Japan Geriatrics Society. Japan Geriatrics Society position statement regarding terminal-stage healthcare management and care for aged individuals. https://www.jpn-geriat-soc.or.jp/tachiba/jgs-tachiba2012.pdf. Accessed 12 May 2021.

27. Japan Geriatrics Society. Guidelines for the decision-making processes in medical and long-term care for the elderly: focusing on the use of artificial hydration and nutrition. https://jpn-geriat-soc.or.jp/proposal/pdf/jgs_ ahn_gl_2012.pdf. Accessed 12 May 2021.

28. Japan Medical Association. Guidelines for physicians' professional ethics. Version 3. Tokyo: Japan Medical Association; 2016.

29. Japanese Society of Nephrology, Japanese Society for Dialysis Therapy, Japanese Society for Transplantation, Japanese Society for Clinical Renal Transplantation, Japanese Society for Peritoneal Dialysis. Treatment options for kidney failure and the current status. 2020. https://jsn.or.jp/ jsn_new/iryou/kaiin/free/primers/pdf/2020allpage.pdf. Accessed 20 May 2021.

30. Warwick G, Mooney A, Russon L, Hardy R. Clinical practice guideline: planning, initiating and withdrawal of renal replacement therapy. UK Renal Association 6th edition final version. Bristol: The Renal Association; 2017.

31. Japanese Society of Nephrology. Clinical practice guidebook for diagnosis and treatment of chronic kidney disease 2012. Tokyo: Tokyo Igaku Sha; 2012.

32. Eneanya ND, Goff SL, Martinez T, Gutierrez N, Klingensmith J, Griffith JL, et al. Shared decision-making in end-stage renal disease: a protocol for a multi-center study of a communication intervention to improve end-oflife care for dialysis patients. BMC Palliat Care. 2015;14:30.

33. Agency for Healthcare Research and Quality. SHARE approach workshop curriculum. https://www.ahrq.gov/health-literacy/curriculum-tools/share ddecisionmaking/workshop/module1/shareworkshop-mod1slides.html\# s8. Accessed 12 May 2021

34. Sudore RL, Lum HD, You JJ, Hanson LC, Meier DE, Pantilat SZ, et al. Defining advance care planning for adults: a consensus definition from a multidisciplinary delphi panel. J Pain Symptom Manag. 2017;53:821-32.e1.
35. Ministry of Health, Labour and Welfare. "Life meeting" (Jjinsei kaigi) decided as the nickname for advance care planning. 2018. https://www. mhlw.go.jp/stf/newpage_02615.html. Accessed 12 May 2021.

36. The Japan Geriatrics Society. Proposal for the promotion of advance care planning. 2019. https://www.jpn-geriat-soc.or.jp/proposal/acp.html. Accessed 12 May 2021.

37. Seymour J, Horne G. ACP facilitating a process of shared decision making In: Thomas K, Lobo B, editors. Advance care planning in end of life care. Oxford: Oxford University Press; 2011. p. 20.

38. Ministry of Health, Labour and Welfare. Guidelines regarding support for individuals who have difficulties in decision making on matters about hospitalization and healthcare. https://www.mhlw.go.jp/content/00051 6181.pdf. Accessed 12 May 2021.

39. Ministry of Health, Labour and Welfare. Commentary on the guidelines for the decision-making process in terminal-stage healthcare: review panel on the optimal decision-making process in terminal-stage healthcare. 2007. https://www.mhlw.go.jp/shingi/2007/05/dl/s0521-11b.pdf. Accessed 12 May 2021.

40. Kawaguchi Y, Nihei H, Hirasawa Y, et al. Study on the formulation of the guidelines for the initiation of dialysis. Fiscal year 1991. In: Ministry of Health, Labour and Welfare Grants, editors. Project report on healthcare for renal failure (group leader: Nobuhide Mimura). Chiba: National Sakura Hospital; 1992. p. 125-32.

41. The Japanese Society for Dialysis Therapy. 2009 guidelines for peritoneal dialysis. J Jpn Soc Dial Ther. 2009;42:285-315.

42. The Japanese Society for Dialysis Therapy. Guidelines for maintenance hemodialysis: hemodialysis initiation. J Jpn Soc Dial Ther. 2013;46:1107-55

43. Okada K, Imada A, Kaizu K, et al. Questionnaire survey of nephrologists: withdrawal from hemodialysis in a patient in the terminal stage of malignancy. J Jpn Soc Dial Ther. 2003;36:1315-26.

44. Okada K, Abe M, Yoshida Y, et al. "Life with dignity" for terminal stage dialysis patients with severe dementia in Japan: a new concept. Nihon Univ J Med. 2009;51:25-34.

45. World Health Organization. WHO definition of palliative care. http://www. who.int/cancer/palliative/definition/en/. Accessed 12 May 2021.

46. Bull R, Youl L, Robertson IK, Mace R, Challenor S, Fassett RG. Pathways to palliative care for patients with chronic kidney disease. J Rent Care. 2014;40:64-73.

47. Izumi S, Nagae H, Sakurai C, Imamura E. Defining end-of-life care from perspectives of nursing ethics. Nurs Ethics. 2012;19:608-18.

48. Japan Pediatric Society: Pediatric Terminal Medical Care Guideline Working Group Ethics Committee. Guidelines for communication of medical policy on the children suffering from serious diseases. 2012. https://www. jpeds.or.jp/modules/guidelines/index.php?content_id=31. Accessed 12 May 2021.

49. Japanese Society for Dialysis Therapy. Shared decision making for the initiation and continuation of dialysis: a proposal from the Japanese society for dialysis therapy. J Jpn Soc Dial Ther. 2020;53:173-217.

\section{Publisher's Note}

Springer Nature remains neutral with regard to jurisdictional claims in published maps and institutional affiliations.

Ready to submit your research? Choose BMC and benefit from

- fast, convenient online submission

- thorough peer review by experienced researchers in your field

- rapid publication on acceptance

- support for research data, including large and complex data types

- gold Open Access which fosters wider collaboration and increased citations

- maximum visibility for your research: over $100 \mathrm{M}$ website views per year

At BMC, research is always in progress.

Learn more biomedcentral.com/submissions 